Article

\title{
Room-Temperature Plastic Deformation of Strontium Titanate Crystals Grown from Different Chemical Compositions
}

\author{
Atsutomo Nakamura ${ }^{1, *}$ (D), Kensuke Yasufuku ${ }^{1}$, Yuho Furushima ${ }^{1}$, Kazuaki Toyoura ${ }^{2}$, \\ K. Peter D. Lagerlöf ${ }^{3}$ and Katsuyuki Matsunaga ${ }^{1,4}$ \\ 1 Department of Materials Physics, Nagoya University, Furo-cho, Chikusa-ku, Nagoya 464-8603, Japan; \\ keeenb9lox@gmail.com (K.Y.); furushima.yuuho@b.mbox.nagoya-u.ac.jp (Y.F.); \\ kmatsunaga@nagoya-u.jp (K.M.) \\ 2 Department of Materials Science and Engineering, Kyoto University, Yoshida Honmachi, Sakyo-ku, \\ Kyoto 606-8501, Japan; toyoura.kazuaki.5r@kyoto-u.ac.jp \\ 3 Department of Materials Science and Engineering, Case Western Reserve University, Cleveland, OH 44106, \\ USA; peter.lagerlof@case.edu \\ 4 Nanostructures Research Laboratory, Japan Fine Ceramics Center, 2-4-1, Mutsuno, Atsuta-ku, \\ Nagoya 456-8587, Japan \\ * Correspondence: anaka@nagoya-u.jp; Tel.: +81-52-789-3366
}

Academic Editor: Helmut Cölfen

Received: 11 October 2017; Accepted: 21 November 2017; Published: 22 November 2017

\begin{abstract}
Oxide materials have the potential to exhibit superior mechanical properties in terms of high yield point, high melting point, and high chemical stability. Despite this, they are not widely used as a structural material due to their brittle nature. However, this study shows enhanced room-temperature plasticity of strontium titanate $\left(\mathrm{SrTiO}_{3}\right)$ crystals through the control of the chemical composition. It is shown that the deformation behavior of $\mathrm{SrTiO}_{3}$ crystals at room temperature depends on the $\mathrm{Sr} / \mathrm{Ti}$ ratio. It was found that flow stresses in deforming $\mathrm{SrTiO}_{3}$ crystals grown from a powder with the particular ratio of $\mathrm{Sr} / \mathrm{Ti}=1.04$ are almost independent of the strain rate because of the high mobility of dislocations in such crystals. As a result, the $\mathrm{SrTiO}_{3}$ crystals can deform by dislocation slip up to a strain of more than $10 \%$, even at a very high strain rate of $10 \%$ per second. It is thus demonstrated that $\mathrm{SrTiO}_{3}$ crystals can exhibit excellent plasticity when chemical composition in the crystal is properly controlled.
\end{abstract}

Keywords: strontium titanate (STO); dislocations; transmission electron microscopy (TEM); single crystals; oxides

\section{Introduction}

Oxide crystals are typically brittle at low temperatures because of their predominant ionic and/or covalent bonding. It is known that slip systems that can be activated in ionic crystals are limited by electrostatic interactions between ions [1], whereas slip system activation in covalent crystals is affected by strongly localized and directional bonds that share electrons [2]. In addition, oxide crystals have large unit cells, leading to large lattice constants, since an oxide is a compound between one or more metallic elements and oxygen atoms. As a result, oxide crystals have large Burgers vectors, which represent the magnitude and direction of dislocations that produce plastic deformation. The larger Burgers vectors make it more difficult to move dislocations. Since the stresses required for dislocation motion are often larger than stresses required for crack propagation, oxide crystals are typically brittle by nature. 
The mechanical properties of strontium titanate $\left(\mathrm{SrTiO}_{3}\right)$ crystals have received growing interest due to the fact that they can exhibit a large degree of plasticity even at low temperatures [3-11]. $\mathrm{SrTiO}_{3}$ is an oxide with a cubic perovskite structure belonging to the $P m \overline{3} m$ space group. It has been reported that $\mathrm{SrTiO}_{3}$ crystals can plastically deform in temperature ranges from $78 \mathrm{~K}$ to $1050 \mathrm{~K}[3,4]$. In addition, $\mathrm{SrTiO}_{3}$ crystals can deform up to and over $10 \%$ if they deform at a slow strain rate of $10^{-4}\left(\mathrm{~s}^{-1}\right)[6,7]$. Recently, the deformation behavior and dislocation structure have been reported to be temperature dependent [9]. In addition, Kondo et al. demonstrated using in-situ observation in transmission electron microscopy (TEM) that dislocation glide can take place in $\mathrm{SrTiO}_{3}$ crystals even at room temperature [10,11]. Thus, $\mathrm{SrTiO}_{3}$ crystals are believed to exhibit plasticity even at low temperatures due to mobility of dislocations belonging to the $\{110\}<110>$ slip systems. However, there is still little known about any key factor providing the high dislocation mobility of $\mathrm{SrTiO}_{3}$.

In the present study, $\mathrm{SrTiO}_{3}$ single crystals grown from powders with varying $\mathrm{Sr} / \mathrm{Ti}$ ratios were deformed at room temperature. This is believed to be due to the fact that a variety of point defects can be formed in $\mathrm{SrTiO}_{3}$ crystals depending on the specific chemical composition and/or chemical environments around the crystals [12,13]. It is well known that point defects in a crystal can interact strongly with dislocations. In general, point defects act as barriers to dislocation motion and result in work hardening of materials followed by nucleation and growth of cracks, leading to brittle fracture. In fact, both $\mathrm{Sr}$ and $\mathrm{O}$ vacancies have been reported to be present around dislocations in $\mathrm{SrTiO}_{3}$ [14-16], forming a Cottrell atmosphere [1]. However, the influence of point defects on the dislocation mobility in $\mathrm{SrTiO}_{3}$ has not been examined.

\section{Experimental}

$\mathrm{SrTiO}_{3}$ single crystals, grown by the Verneuil method from high purity $\mathrm{SrTiO}_{3}$ powder $(99.9 \mathrm{wt} \%$ and $\mathrm{Sr} / \mathrm{Ti}=1.002)$ and high purity $\mathrm{SrCO}_{3}$ powder $(99.99 \mathrm{wt} \%)$, were used for the present deformation experiments. Typically, $\mathrm{Sr}$ deficiency is induced during crystal growth of $\mathrm{SrTiO}_{3}$ crystals due to the evaporation of $\mathrm{Sr}$ ahead of the solidification front [17]. In addition, $\mathrm{Sr}$ vacancies are easily formed in $\mathrm{SrTiO}_{3}$ because of the low formation energy $[12,13]$. For this reason, single crystals grown with a non-stoichiometric starting powder having excess $\mathrm{Sr}(\mathrm{Sr} / \mathrm{Ti}>1)$ would result in fewer $\mathrm{Sr}$ vacancies in the resultant $\mathrm{SrTiO}_{3}$ crystal. Thus, single crystals grown using starting powders with two composition ratios, $\mathrm{Sr} / \mathrm{Ti}=1.00$ and $\mathrm{Sr} / \mathrm{Ti}=1.04$, respectively, were selected to be used in this study. As the starting powder of $\mathrm{Sr} / \mathrm{Ti}=1.00,1000.00 \mathrm{~g}$ of the high purity $\mathrm{SrTiO}_{3}$ powder was employed as it was. On the other hand, a mixed powder of $1000.00 \mathrm{~g}$ high purity $\mathrm{SrTiO}_{3}$ powder and $32.18 \mathrm{~g}$ high purity $\mathrm{SrCO}_{3}$ powder was employed as the starting powder of $S r / T i=1.04$. The growing of all single crystals was performed using a Verneuil furnace at Shinkosha Co., Ltd. (Yokohama, Japan). Here, we note that, considering the evaporation loss of $\mathrm{Sr}$, excess $\mathrm{Sr}$ does not necessarily exist even in the crystals grown from the powder with $\mathrm{Sr} / \mathrm{Ti}=1.04$. Meanwhile, it is difficult to display the real atomic ratios of $S r / T i$ in the grown crystals because a slight change in the ratios is buried in a mass of $\mathrm{Sr}$ and $\mathrm{Ti}$ atoms. The grown crystals from $\mathrm{Sr} / \mathrm{Ti}=1.04$ should have fewer $\mathrm{Sr}$ vacancies than those from $\mathrm{Sr} / \mathrm{Ti}=1.00$.

Figure 1 shows the shapes and crystallographic orientation of the specimens for deformation tests. Specimens were deformed by compression along [001] at strain rates of $\dot{\varepsilon}=1.0 \times 10^{-5}\left(\mathrm{~s}^{-1}\right)$ and $\dot{\varepsilon}=1.0 \times 10^{-1}\left(\mathrm{~s}^{-1}\right)$ in air at $297 \mathrm{~K}$. Note that $\dot{\varepsilon}=1.0 \times 10^{-1}\left(\mathrm{~s}^{-1}\right)$ is a high strain rate because specimens must change their length by $10 \%$ every second. In the case of the deformation along

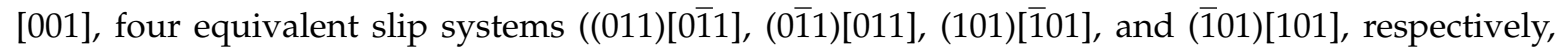
which are illustrated in Figure 1) are expected to be primary slip systems because they all have a Schmid factor equal to 0.5. During plastic deformation, the specimen's length and shape were recorded using a high-speed optical camera in order to confirm slip lines formed on their polished surfaces. The deformed crystals were examined by TEM (Hitachi H- $800,200 \mathrm{kV}$ ) to identify the density and type of dislocations that were introduced during the plastic deformation. Thin foils for TEM observations were prepared by a standard technique. That is, the specimens for deformation were mechanically sliced and ground to a thickness of about $60 \mu \mathrm{m}$ and then attached with a stainless steel 
single hole mesh for reinforcement. The center of the foils was milled by an Ar ion beam using an ion polishing system (Gatan PIPS II) to obtain electron transparency.
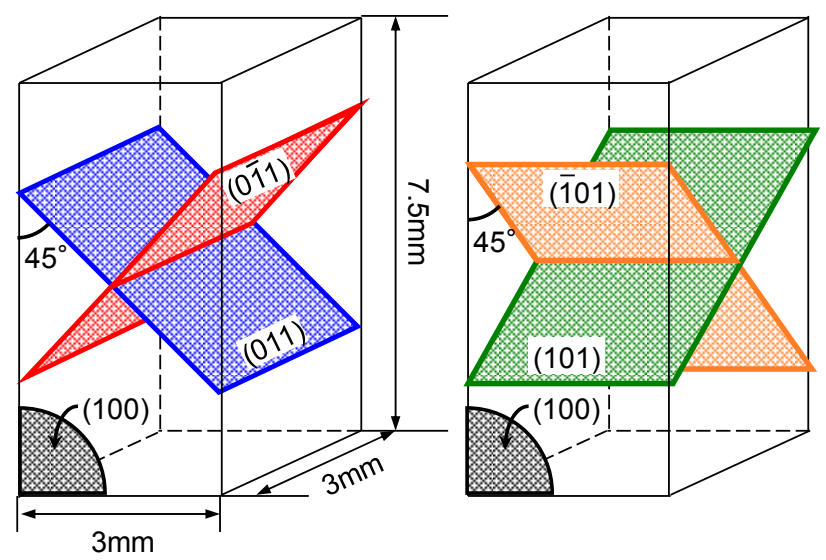

Figure 1. Schematic illustrations showing specimen shapes and available slip systems in the present deformation tests.

\section{Results and Discussion}

Figure 2a,b show the stress-strain curves that were obtained for the specimens with the two different chemical compositions of $\mathrm{Sr} / \mathrm{Ti}=1.00$ (Figure 2a) and $\mathrm{Sr} / \mathrm{Ti}=1.04$ (Figure $2 \mathrm{~b}$ ) as starting powders, respectively. It can be seen that stresses in all the curves gradually increase with increasing strain after yielding, and the deformed specimens give rise to brittle failure after a significant amount of work hardening. It was found that the $S r / T i=1.04$ specimens exhibit lower yield and flow stresses as well as larger fracture strain, compared with the $\mathrm{Sr} / \mathrm{Ti}=1.00$ specimens. The yield stresses, flow stresses (at a plastic strain of $\varepsilon_{\mathrm{p}}=3 \%$ ), and fracture strains of each specimen are shown in Table 1 . As can be seen from the flow stresses at $\varepsilon_{\mathrm{p}}=3 \%$, the stress for $S r / T i=1.04$ at $\dot{\varepsilon}=1.0 \times 10^{-1}\left(\mathrm{~s}^{-1}\right)$ is higher by only $20 \%$ compared with the specimen deformed at $\dot{\varepsilon}=1.0 \times 10^{-5}\left(\mathrm{~s}^{-1}\right)$, in spite of the fact that the strain rate is $10^{4}$ times higher. In contrast, the stress for $S r / T i=1.00$ increases by $31 \%$ with respect to the higher strain rate. The fracture stresses for the $S r / T i=1.04$ specimens are significantly higher than those for the $S r / T i=1.00$ specimens.

Table 1. Deformation stresses and fracture strains for $\mathrm{SrTiO}_{3}$ single crystals.

\begin{tabular}{ccccc}
\hline Sr/Ti & Strain Rate $\left(\mathbf{s}^{-\mathbf{1}}\right)$ & Yield Stress $\mathbf{( M P a )}$ & Flow Stress (at $\left.\boldsymbol{\varepsilon}_{p}=\mathbf{3 \%}\right) \mathbf{( M P a )}$ & Fracture Strain (\%) \\
\hline \multirow{2}{*}{1.04} & $1.0 \times 10^{-5}$ & 112 & 122 & 13.6 \\
& $1.0 \times 10^{-1}$ & 135 & 147 & 13.1 \\
\hline \multirow{2}{*}{1.00} & $1.0 \times 10^{-5}$ & 119 & 138 & 7.4 \\
& $1.0 \times 10^{-1}$ & 160 & 180 & 8.5 \\
\hline
\end{tabular}

Figure $3 \mathrm{a}, \mathrm{b}$ show shapes and surface morphologies of the $\mathrm{Sr} / \mathrm{Ti}=1.00$ and $\mathrm{Sr} / \mathrm{Ti}=1.04$ specimens deformed at $\dot{\varepsilon}=1.0 \times 10^{-1}\left(\mathrm{~s}^{-1}\right)$, respectively. In both specimen types, slip lines become evident at around $\varepsilon_{\mathrm{p}}=3 \%$ and continue to be generated until failure. Thus, it is evident that slip lines are formed even at the high strain rate of $\dot{\varepsilon}=1.0 \times 10^{-1}\left(\mathrm{~s}^{-1}\right)$, indicating prominent multiplication and glide motion of dislocations, although the $S r / T i=1.00$ specimen fails at an earlier stage of deformation. These results are similar to those observed in ordinary single crystals deformed at a slower strain rate. It should be mentioned that the formed slip lines originate from two particular primary slip systems,

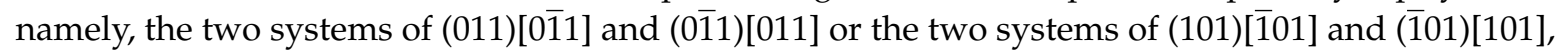
although the Schmid factors for all four of the slip systems are equal. This means that either the two

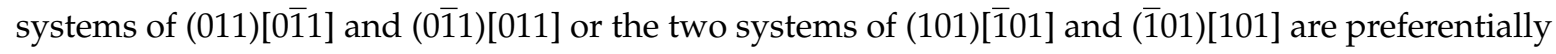


activated during plastic deformation. As a result, only the horizontal slip lines were observed on a side surface of deformed specimens, as shown in Figure 3. This tendency is consistent with the previous reports [6,9] of plastic deformation of $\mathrm{SrTiO}_{3}$ crystals.
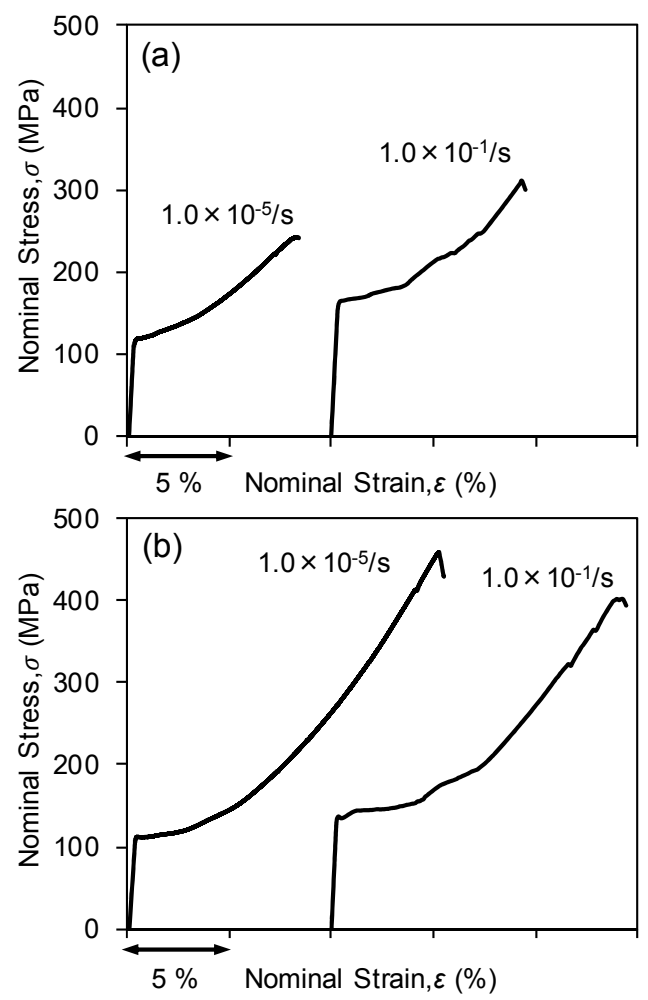

Figure 2. Stress-strain curves of $\mathrm{SrTiO}_{3}$ single crystals with different chemical compositions, deformed at constant strain rates of $\dot{\varepsilon}=1.0 \times 10^{-5}\left(\mathrm{~s}^{-1}\right)$ and $\dot{\varepsilon}=1.0 \times 10^{-1}\left(\mathrm{~s}^{-1}\right)$. (a) $\mathrm{Sr} / \mathrm{Ti}=1.00,(\mathbf{b}) \mathrm{Sr} / \mathrm{Ti}=1.04$ as the starting powders.

\section{(a) From $\mathrm{Sr} / \mathrm{Ti}=1.00$}
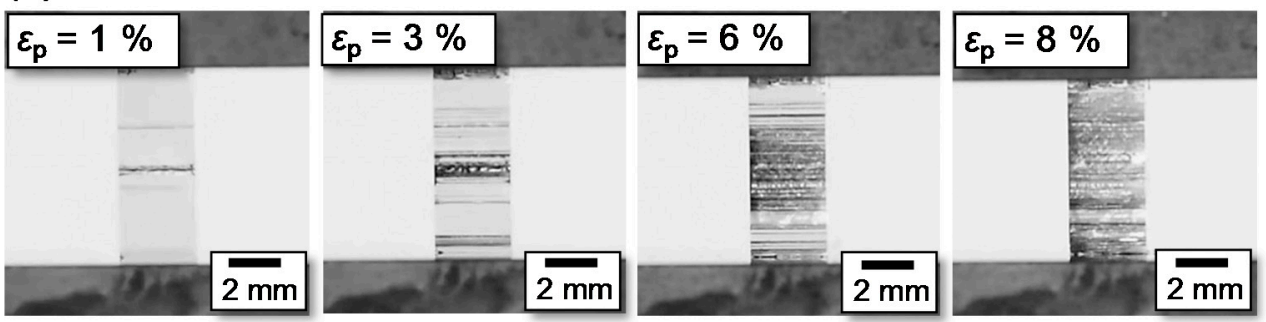

\section{(b) From $\mathrm{Sr} / \mathrm{Ti}=1.04$}
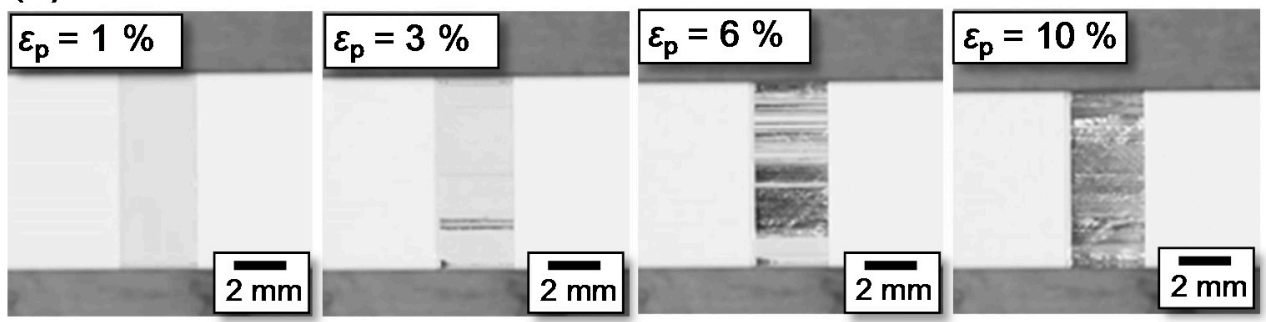

Figure 3. Shapes and surface morphologies of the deformed specimens. (a) The $S r / T i=1.00$ specimen deformed at $\dot{\varepsilon}=1.0 \times 10^{-1}\left(\mathrm{~s}^{-1}\right)$. (b) The $S r / T i=1.04$ specimen deformed at $\dot{\varepsilon}=1.0 \times 10^{-1}\left(\mathrm{~s}^{-1}\right)$. Here, each $\varepsilon_{\mathrm{p}}$ in the figures indicates the plastic strain. 
Figure 4a,b show typical TEM bright field images taken from a $\mathrm{Sr} / \mathrm{Ti}=1.04$ specimen deformed at $\dot{\varepsilon}=1.0 \times 10^{-1}\left(\mathrm{~s}^{-1}\right)$ up to $\varepsilon_{\mathrm{p}}=3 \%$. The incident beam direction is normal to the (011) primary slip plane, where the observed foil for TEM was prepared parallel to the slip lines formed by a primary slip system of (011)[011]. It can be seen that many dislocations are introduced by the deformation, and most of the dislocations are present parallel to the (011) primary slip plane. Since glide dislocations in $\mathrm{SrTiO}_{3}$ have Burgers vectors of the $1 / 2<011>$ type, the Burgers vectors of the dislocations in deformed specimens should be $\pm 1 / 2[0 \overline{1} 1]$ according to activation of the (011)[01] slip system. The image in Figure $4 \mathrm{~b}$ is obtained from the same field as Figure $4 \mathrm{a}$ by using a different diffraction vector of $g=100$. It can be seen that contrasts from almost all dislocations disappear under the diffraction vector of $g=100$. This is because the $\pm 1 / 2[0 \overline{1} 1]$ dislocations originating from the (011)[011] slip system should lose contrasts according to the $\boldsymbol{g} \cdot \boldsymbol{b}=0$ condition [18]. Thus, the dislocation substructure formed by a deformation test is believed to be mainly formed due to the activation of a primary slip system.

Figure 5 represents the dislocation substructure of a $\mathrm{Sr} / \mathrm{Ti}=1.04$ specimen deformed at $\dot{\varepsilon}=1.0 \times 10^{-5}\left(\mathrm{~s}^{-1}\right)$, which is also observed normally to the (011) primary slip plane. It can be also seen from the images that the density and type of introduced dislocations are almost same as those in the specimen deformed at $\dot{\varepsilon}=1.0 \times 10^{-1}\left(\mathrm{~s}^{-1}\right)$. It should be noticed from comparison of the dislocation substructures that the density and type of introduced dislocations are very similar in spite of the great difference in the given strain rates. Additionally, dislocation densities of the specimens deformed up to $\varepsilon_{\mathrm{p}}=3 \%$ were about $1 \times 10^{13} / \mathrm{m}^{2}$, estimated using the Keh method [19] from the TEM images, regardless of given strain rates. The obtained dislocation densities are consistent with the values in earlier research [9], where the densities of dislocations in the un-deformed specimen and the specimen deformed up to $1 \%$ plastic strain at $25^{\circ} \mathrm{C}$ were reported to be $3.7 \times 10^{12} / \mathrm{m}^{2}$ and $7.9 \times 10^{12} / \mathrm{m}^{2}$, respectively. In fact, since the difference in flow stresses is not great, as can be seen from the stresses at $\varepsilon_{\mathrm{p}}=3 \%$ in Table 1, then it would be reasonable to conclude that dislocation densities were almost independent of given strain rates. Meanwhile, the image in Figure 6 shows the dislocation substructure of a $S r / T i=1.00$ specimen deformed at $\dot{\varepsilon}=1.0 \times 10^{-5}\left(\mathrm{~s}^{-1}\right)$. The density and type of introduced dislocations are also similar, regardless of the difference in chemical compositions as starting powders. Consequently, it can be said that the ratio of $\mathrm{Sr} / \mathrm{Ti}$ does not strongly affect the dislocation substructure.

From the above discussion, it has been shown that the yield and flow stresses become lower in the $S r / T i=1.04$ specimens without changing the character of the dislocation substructures. The $S r / T i$ $=1.00$ specimens are believed to have more point defects, such as $\mathrm{Sr}$ vacancies and Ti anti-sites that substitute for Sr, since Sr deficiency should be induced through $\mathrm{Sr}$ evaporation during crystal growth. Conversely, it is plausible that the $\mathrm{Sr} / \mathrm{Ti}=1.04$ specimens have a composition that is closer to the stoichiometric composition in a grown crystal. A more stoichiometric composition in the grown crystal will give rise to fewer point defects, resulting in less interaction with moving dislocations. This may be the reason why the $\mathrm{Sr} / \mathrm{Ti}=1.04$ specimens exhibit lower yield and flow stresses. 

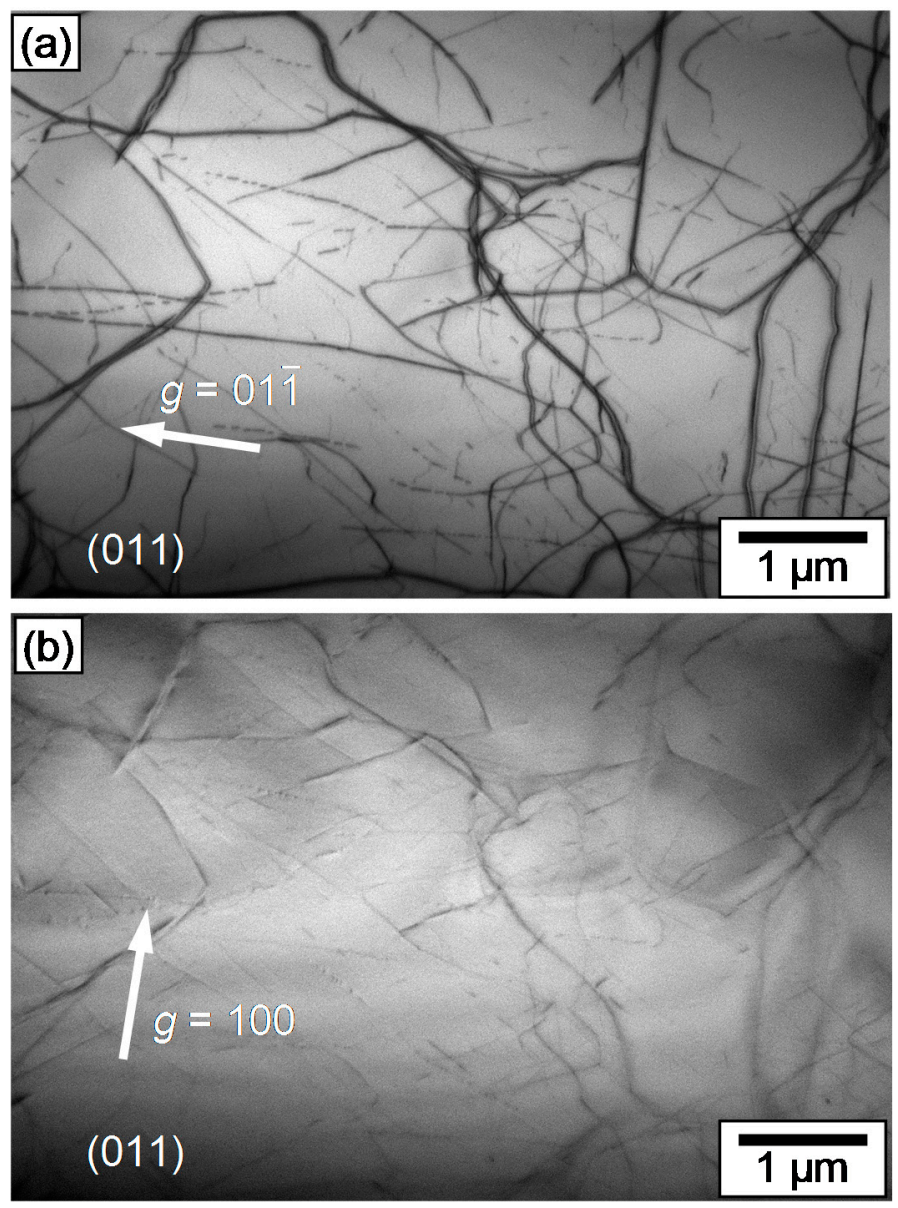

Figure 4. Typical TEM bright field images taken from the $S r / T i=1.04$ specimen deformed at $\dot{\varepsilon}=1.0 \times 10^{-1}\left(\mathrm{~s}^{-1}\right)$ up to $\varepsilon_{\mathrm{p}}=3 \%$. The image in (b) was obtained from the same field as in (a) using a different diffraction vector.

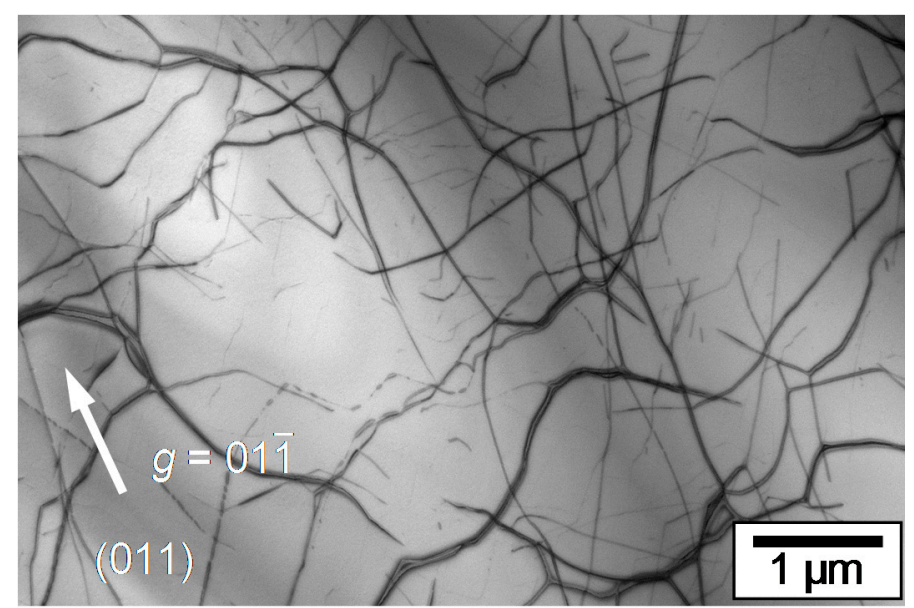

Figure 5. A typical TEM bright field image taken from the $S r / T i=1.04$ specimen deformed at $\dot{\varepsilon}=1.0 \times 10^{-5}\left(\mathrm{~s}^{-1}\right)$ up to $\varepsilon_{\mathrm{p}}=3 \%$. 


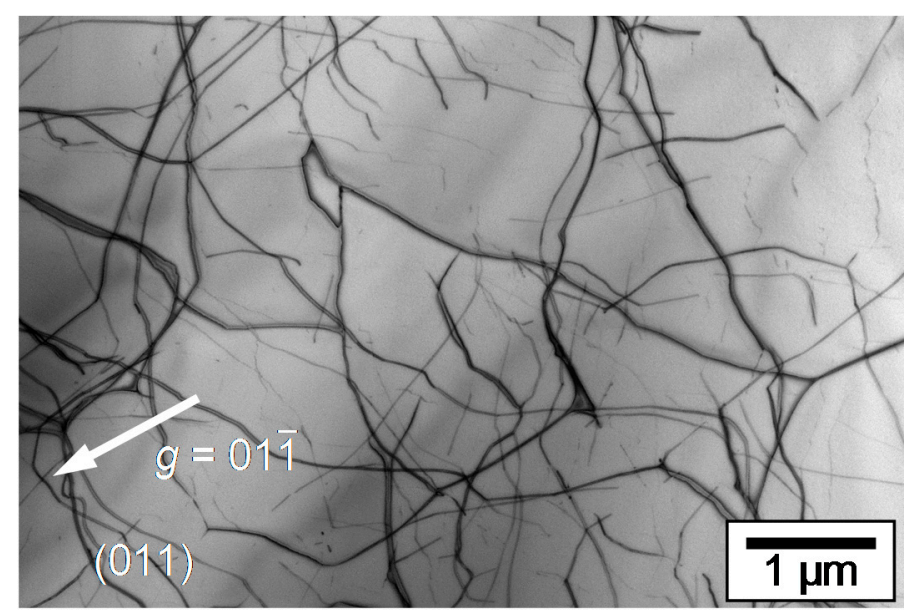

Figure 6. A typical TEM bright field image taken from the $S r / T i=1.00$ specimen deformed at $\dot{\varepsilon}=1.0 \times 10^{-5}\left(\mathrm{~s}^{-1}\right)$ up to $\varepsilon_{\mathrm{p}}=3 \%$.

It is interesting to note that strain-rate dependency of the deformation stress is small for both the $S r / T i=1.00$ and $S r / T i=1.04$ specimens, although the degree is more prominent in the case of $\mathrm{Sr} / \mathrm{Ti}=1.04$. In order to evaluate the strain-rate dependency with respect to dislocation velocity in these specimens, an analysis will be done based on the Orowan relationship [1],

$$
\dot{\gamma}=\rho b \bar{v}
$$

where $\dot{\gamma}$ is the shear strain rate, $\rho$ is the density of mobile dislocations, $b$ is the magnitude of the Burgers vector of the mobile dislocations, and $\bar{v}$ is the average motion speed of the mobile dislocations. In addition, the relation between $\bar{v}$ and deformation stress is given by

$$
\bar{v} \approx \tau^{m}
$$

where $\tau$ is the shear stress and $m$ is the stress exponent [20,21]. As can be seen from TEM images at $\varepsilon_{\mathrm{p}}=3 \%$ in Figures 4 and 5, the dislocation densities of deformed specimens are similar even after increasing the strain rate by a factor of $10^{4}$. Here, we note that dislocations that are observable in the TEM include both mobile and immobile dislocations, and most of the observable dislocations in some cases can be immobile [22]. Accordingly, the densities of mobile dislocations in deforming specimens are still unknown. First of all, we assume that the density of mobile dislocations should be independent of the strain rate as the dislocation substructures introduced by the deformation tests are very similar in spite of great difference in the given strain rates. In this assumption, the dislocation velocity of the mobile dislocations- $\bar{v}$ in Equation (1) - must increase by a factor of $10^{4}$ in the case of the high strain rate of $\dot{\varepsilon}=1.0 \times 10^{-1}\left(\mathrm{~s}^{-1}\right)$ since the strain rate, $\dot{\gamma}$, should be proportional to the dislocation velocity, $\bar{v}$. Moreover, since the flow stresses at $\varepsilon_{\mathrm{p}}=3 \%$ have been determined (see Figure 1 and Table 1) for both cases of $\dot{\varepsilon}=1.0 \times 10^{-5}\left(\mathrm{~s}^{-1}\right)$ and $\dot{\varepsilon}=1.0 \times 10^{-1}\left(\mathrm{~s}^{-1}\right)$, the stress exponent $m$ for $\mathrm{SrTiO}_{3}$ crystals with a particular chemical composition can be estimated by using Equation (2). The values of $m$ in this assumption are calculated to be 35 and 49 for the $\mathrm{Sr} / \mathrm{Ti}=1.00$ and $\mathrm{Sr} / \mathrm{Ti}=1.04$ specimens, respectively. Next, we assume that the density of mobile dislocations depends on the strain rate. Two hypotheses were considered: (i) there are ten times as many mobile dislocations, and (ii) there are one-tenth as many mobile dislocations in the case of the high strain rate of $1.0 \times 10^{-1}\left(\mathrm{~s}^{-1}\right)$. Even in these hypothetical situations, the value of $m$ can be estimated by using Equations (1) and (2). The values of $m$ derived from (i) i.e., the hypothesis of ten times as many mobile dislocations, can be calculated to be 26 and 37 for the $S r / T i=1.00$ and $S r / T i=1.04$ specimens, respectively. Meanwhile, the values of $m$ derived from (ii) i.e., the hypothesis of one-tenth as many mobile dislocations can be 
calculated to be 43 and 62 for the $S r / T i=1.00$ and $S r / T i=1.04$ specimens, respectively. Although the $m$ in Equation (2) mainly depends on deformation flow stresses, the obtained values of $m$ still have a dependency on the densities of mobile dislocations for each of the strain rates. Nevertheless, it is worth pointing out the fact that the stress exponent $m$ for most crystalline materials, including metals, is less than 10 [21]. The $m$ for $\mathrm{SrTiO}_{3}$ would have definitely high values although there is still room for argument about the densities of mobile dislocations. Regardless, it can be said that the dislocation dynamics in $\mathrm{SrTiO}_{3}$ single crystals are remarkable and interesting. We note that a higher value of stress exponent corresponds to smaller strain-rate dependency of deformation flow stresses. In addition, it can be seen from Equation (2) that a larger value of the stress exponent, $m$, results in a higher value of the dislocation velocity of the mobile dislocations, $\bar{v}$, for the same stress level, $\tau$. That is, the dislocations in the $S r / T i=1.04$ specimens would have higher mobility as compared with the dislocations in the $\mathrm{Sr} / \mathrm{Ti}=1.00$ specimens. This is believed to be due to fewer point defects for a $\mathrm{SrTiO}_{3}$ crystal made from a starting powder with $\mathrm{Sr} / \mathrm{Ti}=1.04$.

Finally, the larger fracture stresses for crystals made from a starting powder with $\mathrm{Sr} / \mathrm{Ti}=1.04$ will be considered. As seen from Figure 2, fracture stresses for the $S r / T i=1.04$ specimens are significantly larger than those for the $\mathrm{Sr} / \mathrm{Ti}=1.00$ specimens. As mentioned previously, the mobility of dislocations for the $S r / T i=1.04$ specimens is significantly higher than that for the $S r / T i=1.00$ specimens. The higher dislocation mobility can reduce stress concentrations during plastic deforming since the dislocations can accommodate the applied stress. The larger fracture stresses for the $\mathrm{Sr} / \mathrm{Ti}=1.04$ specimens would arise from the higher dislocation mobility. In contrast, the $S r / T i=1.00$ specimens fail at lower stresses. This can be explained by the relatively lower dislocation mobility, which has a potential to induce higher stress concentrations during plastic deformation.

\section{Conclusions}

From deformation tests of $\mathrm{SrTiO}_{3}$ single crystals, it was found that a crystal grown with the ratio of $\mathrm{Sr} / \mathrm{Ti}=1.04$ as the starting powder can deform with a high degree of plasticity up to a strain of more than $10 \%$ at room temperature, even at a very high strain rate of $\dot{\varepsilon}=0.1\left(\mathrm{~s}^{-1}\right)$. It is remarkable that the strain-rate dependency of deformation flow stress in $\mathrm{SrTiO}_{3}$ is rather small, in particular for crystals made from powders with $\mathrm{Sr} / \mathrm{Ti}=1.04$, meaning that dislocations exhibit a relatively high mobility in these crystals. As a result, larger fracture stresses and strains could be attained for the $\mathrm{Sr} / \mathrm{Ti}=1.04$ specimens. Here, it should be also mentioned that it is still unclear whether the crystals grown with the ratio of $\mathrm{Sr} / \mathrm{Ti}=1.04$ exhibit the highest plasticity or not. Namely, there is a possibility that a better composition of powders for higher plasticity of $\mathrm{SrTiO}_{3}$ crystals will be found in the future. It is likely that the crystals grown from $\mathrm{Sr} / \mathrm{Ti}=1.04$ should have fewer $\mathrm{Sr}$ vacancies than those from $\mathrm{Sr} / \mathrm{Ti}=1.00$, since additional $\mathrm{Sr}$ elements are mixed with the starting powder. Moreover, the crystals from $\mathrm{Sr} / \mathrm{Ti}=1.04$ exhibited higher plasticity as an actual fact. It can be said that the plasticity of $\mathrm{SrTiO}_{3}$ crystals could be enhanced if the chemical composition is properly controlled so as to decrease point defects in a crystal. This suggests that it may be possible to enhance the plasticity of other oxide materials by controlling the chemical composition and resulting point defect populations.

Acknowledgments: The authors gratefully acknowledge the financial support by a Grant-in-Aid for Scientific Research on Innovative Areas "Nano Informatics" (JP25106002) from Japan Society for the Promotion of Science (JSPS). A part of this study was supported by JSPS KAKENHI Grant Numbers JP15H04145, JP16K14414, JP17H06094 and JP17K18983. A.N. also thanks Iketani Science and Technology Foundation for the financial support. Additionally, this work was partly supported by Nanotechnology Platform Program (Advanced Characterization Nanotechnology Platforms of Nagoya Univ.) of the Ministry of Education, Culture, Sports, Science and Technology (MEXT), Japan.

Author Contributions: Atsutomo Nakamura and Katsuyuki Matsunaga conceived the research idea, and Atsutomo Nakamura designed the experiments; Kensuke Yasufuku, Yuho Furushima, and Atsutomo Nakamura performed the experiments and analyzed the data; K. Peter D. Lagerlöf, Kazuaki Toyoura, and Katsuyuki Matsunaga advised the experiments; all authors discussed the results and wrote the paper.

Conflicts of Interest: The authors declare no competing financial interests. 


\section{References}

1. Hirth, J.P.; Lothe, L. Theory of Dislocations, 2nd ed.; Wiley: New York, NY, USA, 1982.

2. Hull, D.; Bacon, D.J. Introduction to Dislocations; Elsevier: Oxford, UK, 2011; pp. 124-133.

3. Brunner, D.; Taeri-Baghbadrani, S.; Sigle, W.; Ruhle, M. Surprising Results of a Study on the Plasticity in Strontium Titanate. J. Am. Ceram. Soc. 2001, 84, 1161-1163. [CrossRef]

4. Gumbsch, P.; Taeri-Baghbadrani, S.; Brunner, D.; Sigle, W.; Ruhle, M. Plasticity and an inverse brittle-to-ductile transition in strontium titanate. Phys. Rev. Lett. 2001, 87, 085505. [CrossRef] [PubMed]

5. Taeri, S.; Brunner, D.; Sigle, W.; Rühle, M. Deformation behaviour of strontium titanate between room temperature and $1800 \mathrm{~K}$ under ambient pressure. Z. Metall. 2004, 95, 433-446. [CrossRef]

6. Brunner, D. Low-temperature plasticity and flow-stress behaviour of strontium titanate single crystals. Acta Mater. 2006, 54, 4999-5011. [CrossRef]

7. Sigle, W.; Sarbu, C.; Brunner, D.; Ruhle, M. Dislocations in plastically deformed $\mathrm{SrTiO}_{3}$. Philos. Mag. 2006, 86, 4809-4821. [CrossRef]

8. Yang, K.-H.; Ho, N.-J.; Lu, H.-Y. Plastic Deformation of <001> Single-Crystal $\mathrm{SrTiO}_{3}$ by Compression at Room Temperature. J. Am. Ceram. Soc. 2011, 94, 3104-3111. [CrossRef]

9. Patterson, E.A.; Major, M.; Donner, W.; Durst, K.; Webber, K.G.; Rodel, J. Temperature-Dependent deformation and dislocation density in $\mathrm{SrTiO}_{3}$ (001) single crystals. J. Am. Ceram. Soc. 2016, 99, 3411-3420. [CrossRef]

10. Kondo, S.; Shibata, N.; Mitsuma, T.; Tochigi, E.; Ikuhara, Y. Dynamic observations of dislocation behavior in $\mathrm{SrTiO}_{3}$ by in situ nanoindentation in a transmission electron microscope. Appl. Phys. Lett. 2012, 100, 181906. [CrossRef]

11. Kondo, S.; Mitsuma, T.; Shibata, N.; Ikuhara, Y. Direct observation of individual dislocation interaction processes with grain boundaries. Sci. Adv. 2016, 2, e1501926. [CrossRef] [PubMed]

12. Tanaka, T.; Matsunaga, K.; Ikuhara, Y.; Yamamoto, T. First-principles study on structures and energetics of intrinsic vacancies in $\mathrm{SrTiO}_{3}$. Phys. Rev. B 2003, 68, 205213. [CrossRef]

13. Liu, B.; Cooper, V.; Xu, H.; Xiao, H.; Zhang, Y.; Weber, W. Composition dependent intrinsic defect structures in $\mathrm{SrTiO}_{3}$. Phys. Chem. Chem. Phys. 2014, 16, 15590-15596. [CrossRef] [PubMed]

14. Takehara, K.; Sato, Y.; Tohei, T.; Shibata, N.; Ikuhara, Y. Titanium enrichment and strontium depletion near edge dislocation in strontium titanate [001]/(110) low-angle tilt grain boundary. J. Mater. Sci. 2014, 49, 3962-3969. [CrossRef]

15. Choi, S.Y.; Kim, S.D.; Choi, M.; Lee, H.S.; Ryu, J.; Shibata, N.; Mizoguchi, T.; Tochigi, E.; Yamamoto, T.; Kang, S.J.L. Assessment of strain-generated oxygen vacancies using $\mathrm{SrTiO}_{3}$ bicrystals. Nano Lett. 2015, 15, 4129-4134. [CrossRef] [PubMed]

16. Furushima, Y.; Arakawa, Y.; Nakamura, A.; Tochigi, E.; Matsunaga, K. Nonstoichiometric [012] dislocation in strontium titanate. Acta Mater. 2017, 135, 103-111. [CrossRef]

17. Scheel, H.J.; Bednorz, J.G.; Dill, P. Crystal growth of strontium titanate. Ferroelectrics 1976, 13, 507-509. [CrossRef]

18. Williams, D.B.; Carter, C.B. Transmission Electron Microscopy: A Textbook for Materials Science, 2nd ed.; Springer: New York, NY, USA, 2009.

19. Keh, A.S. Dislocation Arrangement in Alpha Iron during Deformation and Recovery. In Direct Observation of Imperfections in Crystal; Interscience Publishers (Wiley): New York, NY, USA, 1961.

20. Messerschmidt, U. Dislocation Dynamics during Plastic Deformation; Springer: Berlin, Germany, 2010; pp. 73-280.

21. Nadgornyi, E. Dislocation Dynamics and Mechanical Properties of Crystals; Pergamon: Oxford, UK, 1988; pp. 1-536.

22. Bergsröm, Y. A dislocation model for the stress-strain behaviour of polycrystalline $\alpha$-Fe with special emphasis on the variation of the densities of mobile and immobile dislocations. Mater. Sci. Eng. 1970, 5, 193-200. [CrossRef]

(C) 2017 by the authors. Licensee MDPI, Basel, Switzerland. This article is an open access article distributed under the terms and conditions of the Creative Commons Attribution (CC BY) license (http:/ / creativecommons.org/licenses/by/4.0/). 